\title{
ATTRACTORS FOR MAPS WITH FRACTIONAL INVERSE
}

\author{
M. R. FERCHICHI AND I. DJELLIT
}

Received 5 November 2004

In this work, we consider some dynamical properties and specific contact bifurcations of two-dimensional maps having an inverse with vanishing denominator. We introduce new concepts and notions of focal points and prefocal curves which may cause and generate new dynamic phenomena. We put in evidence a link existing between basin bifurcation of a map with fractional inverse and the prefocal curve of this inverse.

\section{Introduction}

Specific class of maps, defined by $x^{\prime}=F(x, y), y^{\prime}=G(x, y)$, with at least one of the components $F$ or $G$ defined by a rational and fractional function, are evidenced in the literature (see the references therein), and have very interesting properties. Some peculiar dynamical properties have been evidenced and observed in iterated maps (or in the inverse) having a vanishing denominator or assuming the form $0 / 0$ in a point of $I R^{2}$. These past years have seen substantial progress on several questions concerning two-dimensional maps. There have been many books on dynamical systems to reflect the recent interest, but relatively few of the books to offer a large account of the area. In particular, there has been a sequence of texts which have gradually developed the subject of bifurcation theory, and have a variety of different strategies to cover the subject, but some areas remained fuzzy.

In this paper, we introduce and characterize new singularities following the notations $[2,3,4,5,6]$, called focal point and prefocal curve, and their role on the geometrical properties of a rational map is described.

A prefocal curve is defined in $[2,3,4,5,6]$ as a set of points for which at least one inverse exists, which focalizes the whole set into a single point, called focal point. A bifurcation of the unstable set of a saddle point (or a saddle cycle), at the contact with the set of points which vanish the denominator (called the set of nondefinition), gives rise to unbounded branches of the unstable set. This kind of bifurcation is specific to maps with denominator.

Another considerable property (for maps with inverse having a vanishing denominator) is a curve, on which the denominator vanishes, which can separate the plane in areas 
characterized by different number of preimages, even though this curve is not a critical curve in the sense of Mira (see [7,8]).

The existence of a focal point of an inverse map may give rise to an attracting particular set, where the focal point has the same behavior of a node with an infinity of invariant curves.

Section 2 is devoted to definitions, properties, and the key role of focal points and prefocal curves. We describe and study the link existing between basin bifurcation of a map with fractional inverse and the prefocal curve of this inverse, and we explain this phenomenon in different terms, making use of Mira's concepts. In Section 3, we explain these concepts in one example: the Bogdanov map.

\section{Definitions and generic properties}

The few definitions and generic properties given here concern maps denoted by $(x, y) \rightarrow$ $\left(x^{\prime}, y^{\prime}\right)=T(x, y)$ of the form given by

$$
T:\left\{\begin{array}{l}
x^{\prime}=F(x, y), \\
y^{\prime}=G(x, y),
\end{array}\right.
$$

where $x$ and $y$ are real variables and at least one of the components has the form of a rational function, that is,

$$
F(x, y)=\frac{N_{1}(x, y)}{D_{1}(x, y)}
$$

and where

$$
G(x, y)=\frac{N_{2}(x, y)}{D_{2}(x, y)} .
$$

In order to simplify, it is assumed that only one of the components has a denominator which can vanish, example $G(x, y)=N(x, y) / D(x, y)$, Thus map (2.1) is written as

$$
T:\left\{\begin{array}{l}
x^{\prime}=F(x, y), \\
y^{\prime}=\frac{N(x, y)}{D(x, y)},
\end{array}\right.
$$

where it is assumed that the functions $F(x, y), N(x, y)$, and $D(x, y)$ are defined in the entire plane $I R^{2}$. The set of nondefinition of $T$ is given by

$$
\delta_{s}=\left\{(x, y) \in I R^{2} \mid D(x, y)=0\right\} .
$$

In the following, one will suppose that $\delta_{s}$ is a smooth curve in the plane. The twodimensional map obtained by successive iteration of $T$ will be well defined, if the initial conditions belong to $E$, given by:

$$
E=I R^{2} \backslash \bigcup_{k=0}^{\infty} T^{-k}\left(\delta_{s}\right) .
$$


In order to define concepts of focal point and prefocal curve, we consider a smooth arc $\gamma$ transverse to $\delta_{s}$ and we study the shape of its image under $T$, that is, $T(\gamma)$. We assume that $\gamma$ is deprived of its point of intersection with $\delta_{s}$.

Definition 2.1. Consider the map (2.1). A point $Q=\left(x_{0}, y_{0}\right)$ is a focal point if at least one component of $T$ takes the form $0 / 0$ in $Q$ and there exist smooth simple $\operatorname{arcs} \gamma(\tau)$, with $\gamma(0)=Q$, such that $\lim _{\tau->0} T(\gamma(\tau))$ is finite. The set of all finite values, obtained in different $\operatorname{arcs} \gamma(\tau)$ through $Q$, is called prefocal curve $\delta_{Q}$.

We can calculate prefocal curve and the focal point analytically by the following method provided that the inverse is known explicitly. We search for the set of $J_{0}^{\prime}$ for which $\operatorname{det}\left(D T^{-1}\right)$ vanishes, and we calculate the images, under $T^{-1}$, points of $J_{0}^{\prime}$. If $J_{0}^{\prime}$ contains a curve $\delta$ such that $T^{-1}(\delta)$ reduces itself to a point $Q$, thus $\delta$ is a prefocal curve for the map $T$, and $Q$ is then the associated focal point.

The proposition which follows shows that $\lim _{\tau->0} T(\gamma(\tau))$ depends only on the slope $m$ at the point $Q$ of the arc $\gamma(\tau)$ and not on $\gamma(\tau)$ itself. The demonstration is in [5].

Proposition 2.2. Let $T$ be a map of the form (2.4) and let $Q$ be a focal point whose corresponding prefocal curve is $\delta_{Q}$. Then,

(1) there exists a one-to-one correspondence between the slope $m$ of an arc $\gamma$ passing through $Q$, not tangent to $\delta_{s}$, and the point $\left(F(Q), y=\lim _{\tau->0} G(\gamma(\tau))\right)$ in which $T(\gamma)$ crosses $\delta_{Q}$,

(2) this correspondence is defined by

$$
\begin{gathered}
m \longrightarrow(F(Q), y(m))=\left(F(Q), \frac{N_{x}^{\prime}+m N_{y}^{\prime}}{D_{x}^{\prime}+m D_{y}^{\prime}}\right), \\
(F(Q), y) \longrightarrow m(y)=\frac{D_{x}^{\prime} y-N_{x}^{\prime}}{N_{y}^{\prime}-D_{y}^{\prime} y}
\end{gathered}
$$

where $N_{x}^{\prime}=\partial N / \partial x\left(x_{0}, y_{0}\right)$ and analogously for the other partial derivatives.

We need to locate geometrically the focal point in the plane. This concept is stated and proved in the following proposition and proof.

Proposition 2.3. Let $T=(F(x, y), G(x, y))$ be a polynomial map of $I R^{2}->I R^{2}$ and let $T^{-1}=(H(x, y), N(x, y) / D(x, y))$ be its inverse. If $T\left(\delta_{s}\right)$ crosses transversely $\delta_{s}$ in a point $Q=\left(x_{0}, y_{0}\right)$, where $\delta_{s}$ is the set of nondefinition of $T^{-1}$, then $Q$ is a focal point of $T^{-1}$.

Proof. We want to prove that if $Q \in \delta_{s} \cap T\left(\delta_{s}\right)$, then $Q$ is a focal point of $T^{-1}$. As $Q=$ $\left(x_{0}, y_{0}\right) \in T\left(\delta_{s}\right)$, there exists $\left(x_{-1}, y_{-1}\right) \in \delta_{s}$ such that $\left(x_{0}, y_{0}\right)=T\left(x_{-1}, y_{-1}\right)$.

Assume that in any neighborhood of $\left(x_{-1}, y_{-1}\right)$, the set of nondefinition $\delta_{s}$ can be represented by the parametric equations

$$
\delta_{s}=\left\{\begin{array}{l}
x(\tau)=x_{-1}+\xi_{1} \tau+\xi_{2} \tau^{2}+\cdots \\
y(\tau)=y_{-1}+\eta_{1} \tau+\eta_{2} \tau^{2}+\cdots
\end{array}\right.
$$


In a neighborhood of $\left(x_{-1}, y_{-1}\right), T$ is expressed as

$$
T=\left\{\begin{array}{l}
x^{\prime}=F(x, y)=F\left(x_{-1}, y_{-1}\right)+F_{x}^{\prime}\left(x-x_{-1}\right)+F_{y}^{\prime}\left(y-y_{-1}\right)+O_{1}\left(x-x_{-1}, y-y_{-1}\right), \\
y^{\prime}=G(x, y)=G\left(x_{-1}, y_{-1}\right)+G_{x}^{\prime}\left(x-x_{-1}\right)+G_{y}^{\prime}\left(y-y_{-1}\right)+O_{2}\left(x-x_{-1}, y-y_{-1}\right),
\end{array}\right.
$$

where $O_{1}$ and $O_{2}$ are terms of the second order, these relations are obtained by considering series expansion of the two functions.

Therefore, in the neighborhood of $\left(x_{-1}, y_{-1}\right)$ and as $\left(x_{0}, y_{0}\right)=T\left(x_{-1}, y_{-1}\right)=\left(F\left(x_{-1}\right.\right.$, $\left.\left.y_{-1}\right), G\left(x_{-1}, y_{-1}\right)\right)$,

$$
T\left(\delta_{s}\right)=\left\{\begin{array}{l}
x^{\prime}(\tau)=x_{0}+\left(F_{x}^{\prime} \xi_{1}+F_{y}^{\prime} \eta_{1}\right) \tau+O_{1}(\tau) \\
y^{\prime}(\tau)=y_{0}+\left(G_{x}^{\prime} \xi_{1}+G_{y}^{\prime} \eta_{1}\right) \tau+O_{2}(\tau)
\end{array}\right.
$$

The Taylor expansions of the polynomial functions $N(x, y)$ and $D(x, y)$, at the point $Q=\left(x_{0}, y_{0}\right)$, are written as

$$
\begin{aligned}
& N(x, y)=N\left(x_{0}, y_{0}\right)+N_{x}^{\prime}\left(x-x_{0}\right)+N_{y}^{\prime}\left(y-y_{0}\right)+O_{3}\left(x-x_{0}, y-y_{0}\right), \\
& D(x, y)=D\left(x_{0}, y_{0}\right)+D_{x}^{\prime}\left(x-x_{0}\right)+D_{y}^{\prime}\left(y-y_{0}\right)+O_{4}\left(x-x_{0}, y-y_{0}\right),
\end{aligned}
$$

where $\mathrm{O}_{3}$ and $\mathrm{O}_{4}$ are higher-order terms and $D\left(x_{0}, y_{0}\right)=0$ by hypothesis.

In the neighborhood of $\left(x_{0}, y_{0}\right)$ and along $T\left(\delta_{s}\right)$, we set

$$
T^{-1}(T(x(\tau), y(\tau)))=(x(\tau), y(\tau))
$$

and then

$$
T(x(\tau), y(\tau)) \neq T(x(0), y(0))=T\left(x_{-1}, y_{-1}\right)=\left(x_{0}, y_{0}\right) \in \delta_{s}
$$

which implies that

$$
\begin{aligned}
\lim _{\tau \rightarrow 0} T^{-1}\left(x^{\prime}(\tau), y^{\prime}(\tau)\right) & =\lim _{\tau \rightarrow 0} T^{-1}(T(x(\tau), y(\tau)))=\lim _{\tau \rightarrow 0}(x(\tau), y(\tau)) \\
& =(x(0), y(0))=\left(x_{-1}, y_{-1}\right) .
\end{aligned}
$$

From relations (2.11), (2.12), (2.13), it results that

$$
\begin{aligned}
\lim _{\tau \rightarrow 0} & \frac{N\left(x^{\prime}(\tau), y^{\prime}(\tau)\right)}{D\left(x^{\prime}(\tau), y^{\prime}(\tau)\right)} \\
& =\lim _{\tau \rightarrow 0} \frac{N\left(x_{0}, y_{0}\right)+N_{x}^{\prime}\left(\left(F_{x}^{\prime} \xi_{1}+F_{y}^{\prime} \eta_{1}\right) \tau\right)+N_{y}^{\prime}\left(\left(G_{x}^{\prime} \xi_{1}+G_{y}^{\prime} \eta_{1}\right) \tau\right)+O_{5}(\tau)}{D_{x}^{\prime}\left(\left(F_{x}^{\prime} \xi_{1}+F_{y}^{\prime} \eta_{1}\right) \tau\right)+D_{y}^{\prime}\left(\left(G_{x}^{\prime} \xi_{1}+G_{y}^{\prime} \eta_{1}\right) \tau\right)+O_{6}(\tau)} \\
& =y_{-1}
\end{aligned}
$$

where $\mathrm{O}_{5}$ and $\mathrm{O}_{6}$ are higher-order terms. 


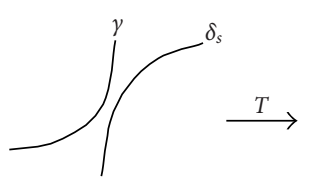

(a)

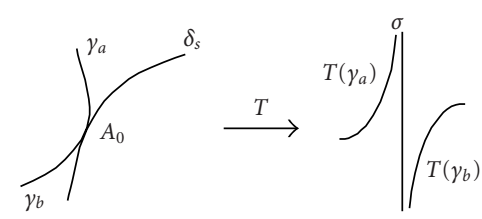

(b)

Figure 3.1. Image of a smooth curve $\gamma$ when there is a contact between this last and the set of nondefinition $\delta_{s}$.

As $y_{-1}$ is finite, then necessarily $N\left(x_{0}, y_{0}\right)=0$, so the limit (2.16) is equal to

$$
\lim _{\tau \rightarrow 0} \frac{N\left(x^{\prime}(\tau), y^{\prime}(\tau)\right)}{D\left(x^{\prime}(\tau), y^{\prime}(\tau)\right)}=\frac{N_{x}^{\prime}\left(F_{x}^{\prime} \xi_{1}+F_{y}^{\prime} \eta_{1}\right)+N_{y}^{\prime}\left(G_{x}^{\prime} \xi_{1}+G_{y}^{\prime} \eta_{1}\right)}{D_{x}^{\prime}\left(F_{x}^{\prime} \xi_{1}+F_{y}^{\prime} \eta_{1}\right)+D_{y}^{\prime}\left(G_{x}^{\prime} \xi_{1}+G_{y}^{\prime} \eta_{1}\right)}=y_{-1} .
$$

It is plain that this limit is finite, since the slope of $\delta_{s}$ in $Q$, which is equal to $-D_{x}^{\prime} / D_{y}^{\prime}$, is different from $T\left(\delta_{s}\right)$ in $Q$, and equal to $\left(G_{x}^{\prime} \xi_{1}+G_{y}^{\prime} \eta_{1}\right) /\left(F_{x}^{\prime} \xi_{1}+F_{y}^{\prime} \eta_{1}\right)$, since we suppose that $T\left(\delta_{s}\right)$ is transverse to $\delta_{s}$ at $Q$.

We have $N(x, y) / D(x, y)$ which takes the form $0 / 0$ in $Q$ and the limit (2.15) is finite, then $Q$ is a focal point focal of $T^{-1}$.

Corollary 2.4. Let $Q$ be a focal point of $T^{-1}$, if $\gamma(\tau)=T\left(\delta_{s}\right)$ and $\gamma(0)=Q$, then $\lim _{\tau->0} T^{-1}(\gamma(\tau))$ belongs to $\delta_{s} \cap \delta_{\mathrm{Q}}$.

We are rather far from a complete and systematic knowledge of the effects of these new concepts. Then we are illustrating our results by a collection of figures.

\section{Geometric properties of focal points and prefocal curves}

In this section, we study how a contact between a segment of the curve $\gamma$ and the set $\delta_{s}$ causes a qualitative noticeable change in the shape of the image $T(\gamma)$, and how a contact of $\gamma$ with a prefocal curve $\delta_{Q}$ causes an important qualitative change in the shape of the preimage $T_{j}^{-1}(\gamma)$.

Consider a segment of the curve $\gamma$ entirely contained in an area where the denominator of the map $T$ does not vanish, and such a map is continuous in every point of $\gamma$. Since $\gamma$ is a compact subset of $I R^{2}$, its image $T(\gamma)$ is also compact. Now imagine that $\gamma$ displaces itself toward $\delta_{s}$, till it becomes tangent at a point $A_{0}=\left(x_{0}, y_{0}\right)$ which is not a focal point. At the contact, $T(\gamma)$ is not more compact and becomes the union of two unbounded branches asymptotic to the line $\sigma$ of equation $x=F\left(x_{0}, y_{0}\right)$ (see Figure 3.1).

Indeed, $T(\gamma)=T\left(\gamma_{a}\right) \cup T\left(\gamma_{b}\right)$, where $\gamma_{a}$ and $\gamma_{b}$ are two parts of $\gamma$ separated from the point $A_{0}=\gamma \cap \delta_{s}$. At the point $A_{0}$, the map $T$ is not defined and the limit of $T(x, y)$ as $(x, y)->A_{0}$ along $\gamma_{a}$ and $\gamma_{b}$ is equal to $\lim _{(x, y)->A_{0}} T(x, y)=\left(F\left(x_{0}, y_{0}\right), \infty\right)$.

In such a situation, any image of $\gamma$ of rank $k>1$, given by $T^{k}(\gamma)$, contains two unbounded disjoint branches asymptotic to the image $T^{k}(\sigma)$ of the line $\sigma$ of rank $k$. 

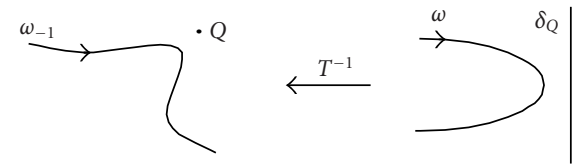

(a)
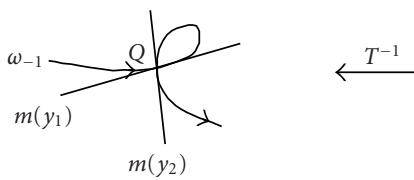

(c)

Figure 3.2. Image under $T^{-1}$ of a smooth curve $\omega$ after crossing through $\delta_{Q}$.

The qualitative change of $T(\gamma)$, due to the contact of $\gamma$ and $\delta_{s}$, as described above, can represent an important contact bifurcation of a rational map $T$ when $\gamma$ is, for example, the local unstable manifold $W^{u}$ of a saddle point or a saddle cycle. In fact, the appearance of an unbounded branch of $W^{u}$, due to the contact with $\delta_{s}$, gives birth to homoclinic points, due to new transversal intersections between unstable and stable sets $W^{s}$ and $W^{u}$, of a same saddle point (or cycle), and which do not come from a tangential contact between $W^{s}$ and $W^{u}$. This implies that, in map with denominator, homoclinic points can be born without causing the occurrence of homoclinic tangency between $W^{s}$ and $W^{u}$, due to a sudden apparition of unbounded branches of $W^{u}$ when it crosses $\delta_{s}$.

Show now the action and the role played by the inverse map on a segment of a smooth curve $\omega$ which tends through a prefocal curve $\delta_{Q}$ until crossing it. It assumes that $\delta_{Q}$ belongs to the line of equation $x=F(Q)$, and that the correspondence defined by $(2.7)$ and (2.8) is verified.

As $\omega$ tends to $\delta_{Q}$, its preimage $\omega_{-1}=T^{-1}(\omega)$ tends through the focal point $Q$. When $\omega$ becomes tangent to $\delta_{Q}$ at a point $C=\left(F(Q), y_{c}\right)$, thus $\omega_{-1}$ is a cuspidal point in $Q$ (Figures 3.2(a) and 3.2(b)). The slope of the common tangent to the two arcs which join each other in $Q$ is given by (2.8).

If the segment of the curve $\omega$ crosses $\delta_{Q}$ in two points $\left(F(Q), y_{1}\right)$ and $\left(F(Q), y_{2}\right)$, thus $\omega_{-1}$ forms a loop of double point to focal point $Q$ (see Figure 3.2). Indeed the images under $T^{-1}$ of the two portions of $\omega$, which cross $\delta_{Q}$, are two $\operatorname{arcs}$ of $\omega_{-1}$ passing through $Q$, and the tangents to these two arcs in a focal point have different slopes, $m\left(y_{1}\right)$ and $m\left(y_{2}\right)$, clarified by formula (2.8).

The following proposition shows the link between the structure of the basin of attraction of an attractor of $T$ and the fact that a prefocal curve being inside or outside the basin. 
Proposition 3.1. Let $T$ be a two-dimensional invertible map whose inverse $T^{-1}$ is with denominator. Let $D_{0}$ be the immediate basin of an attractor $A$ of $T$ and $\delta_{Q}$ a prefocal curve of $T^{-1}$ associated with the focal point $Q$. Suppose that $\delta_{s} \cap D_{0} \neq \varnothing$, where $\delta_{s}$ is the set of nondefinition of $T^{-1}$. The basin $D$ of $A$ is connected if and only if $Q$ belongs to $D$.

Demonstration. First, we have the following implications:

(i) $\delta_{s} \cap D_{0} \neq \varnothing \Rightarrow T\left(\delta_{s}\right) \cap D_{0} \neq \varnothing$,

(ii) $Q \in D_{0} \Leftrightarrow T^{-1}\{Q\}=\delta_{Q} \subset T^{-1}\left(D_{0}\right)$,

(iii) $Q \notin D_{0} \Leftrightarrow \delta_{Q} \cap T^{-1}\left(D_{0}\right)=\varnothing$.

We denote by $D_{0}^{+}$and $D_{0}^{-}$the parts of $D_{0}$ situated, respectively, above and below of $\delta_{s}$. Since $T$ is invertible and $T^{-1}\left(\delta_{s}\right)$ is the set of points at infinity of $T^{-1}$ plus $\delta_{Q}$, then $T^{-1}\left(D_{0}^{+}\right)$and $T^{-1}\left(D_{0}^{-}\right)$are on all sides of $\delta_{Q}$.

(1) We show that if $D$ is connected, then $Q$ belongs to $D$.

Suppose the contrary, this means that $Q \notin D$, which implies that $Q \notin D_{0}$, and thus $\delta_{Q}$ does not intersect $T^{-1}\left(D_{0}\right)=T^{-1}\left(D_{0}^{+} \cup D_{0}^{-}\right)=T^{-1}\left(D_{0}^{+}\right) \cup T^{-1}\left(D_{0}^{-}\right)$. Since it has been shown that $T^{-1}\left(D_{0}^{+}\right)$and $T^{-1}\left(D_{0}^{-}\right)$are on both sides of $\delta_{Q}$, it follows that $T^{-1}\left(D_{0}\right)$ is not connected, and then $D$ is not connected. This is a contradiction, $D$ is thus connected, and therefore $Q \in D$.

(2) To prove that if $Q$ belongs to $D$, then $D$ is connected. We suppose that $Q \in D_{0}$, which implies that $T^{-1}\{Q\}=\delta_{Q} \subset T^{-1}\left(D_{0}\right)=T^{-1}\left(D_{0}^{+}\right) \cup T^{-1}\left(D_{0}^{-}\right)$. Hence $T^{-1}\left(D_{0}^{+}\right)$and $T^{-1}\left(D_{0}^{-}\right)$are on either side of $\delta_{Q}$, therefore, so that $T^{-1}\left(D_{0}\right)$ can contain $\delta_{Q}$, it is necessary that $T^{-1}\left(D_{0}^{+}\right)$and $T^{-1}\left(D_{0}^{-}\right)$merge, this means that $T^{-1}\left(D_{0}^{+}\right) \cap T^{-1}\left(D_{0}^{-}\right)=\delta_{Q}$, it follows that $T^{-1}\left(D_{0}\right)$ is connected, and then $D$ is connected.

\section{Application to Bogdanov map}

The Bogdanov map is defined in the whole plane $I R^{2}$ by the following equations [1]:

$$
T(x, y)=\left\{\begin{array}{l}
x^{\prime}=x+y+a y+b x(x-1)+c x y, \\
y^{\prime}=y+a y+b x(x-1)+c x y,
\end{array}\right.
$$

where $a, b, c$ are real parameters. Bogdanov map is invertible, with one of the components of the inverse $T^{-1}$ having a vanishing denominator. The inverse $T^{-1}$ is given by

$$
T^{-1}\left(x^{\prime}, y^{\prime}\right)=\left\{\begin{array}{l}
x=x^{\prime}-y^{\prime} \\
y=\frac{y^{\prime}-b\left(x^{\prime}-y^{\prime}\right)\left(x^{\prime}-y^{\prime}-1\right)}{1+a+c\left(x^{\prime}-y^{\prime}\right)} .
\end{array}\right.
$$

The set of nondefinition $\delta_{s}$ of $T^{-1}$ is as follows:

$$
y=x+\frac{1+a}{c}
$$




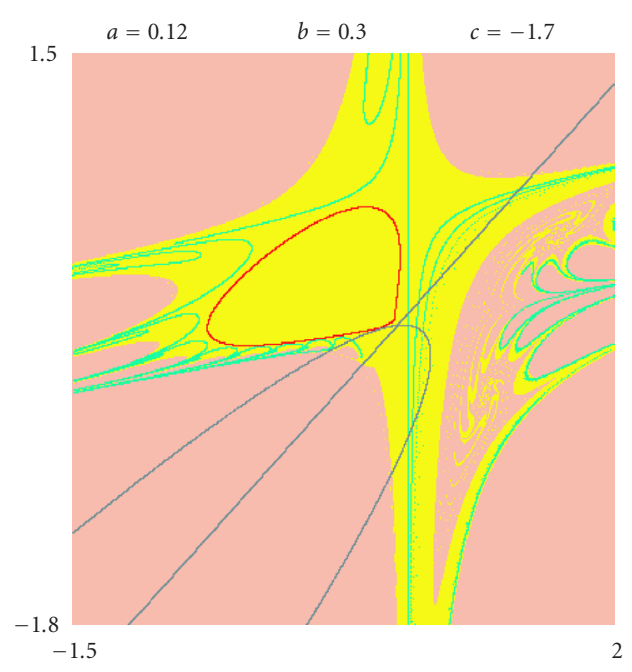

Figure 4.1. The attractor is the closed curve, its basin is colored clear gray.

The Jacobian matrix of $T$ is equal to

$$
D T(x, y)=\left[\begin{array}{cc}
1+2 b x-b+c y & 1+a+c x \\
2 b x-b+c y & 1+a+c x
\end{array}\right]
$$

The Jacobian is

$$
\operatorname{det} D T(x, y)=1+a+c x
$$

which vanishes on the curve of equation given by $x=(-1-a) / c$. The image of this curve is reduced to a point

$$
\left(\frac{-1-a}{c}+b\left(\frac{-1-a}{c}\right)\left(\frac{-1-a}{c}-1\right), b\left(\frac{-1-a}{c}\right)\left(\frac{-1-a}{c}-1\right)\right) .
$$

Consequently, this point is a focal point of $T^{-1}$ and the curve of equation $x=(-1-a) / c$ is the associated prefocal curve.

(I) One Fixes $b=0.3$ and $c=-1.7$ and one varies the parameter $a$.

(1) For $a=0.12$ (see Figure 4.1), the map $T$ has an attractor, which is a closed invariant curve resulting from a Neimark-Hopf bifurcation. The focal point of $T^{-1}$ is the intersection point of $\delta_{s}$ and $T\left(\delta_{s}\right)$, and inside the basin of attraction of the invariant closed curve. Hence, the corresponding prefocal curve and its images under $T^{-1}$ are inside the basin of attraction. That's which gives an unbounded basin with a boundary asymptotic to the prefocal curve and its images under $T^{-1}$. We remark that the attractor does not touch 


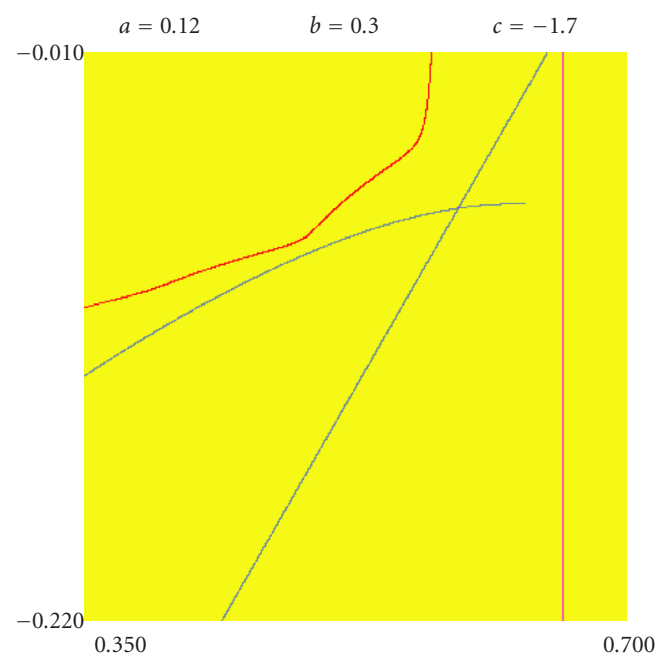

Figure 4.2. Enlargement of a portion of Figure 4.1.

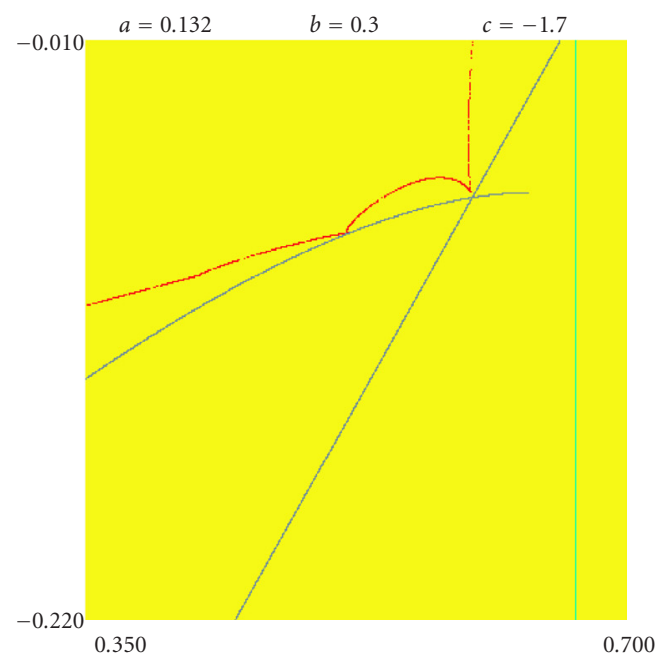

Figure 4.3. Creation of cusp points on the attractor, contact the cusp and the focal point.

the prefocal curve and then the focal point. An enlargement of a portion of the attractor, close to the prefocal curve, is illustrated by Figure 4.2.

(2) We fix $a=0.132$, and the attractor comes closer to the focal point, a part situated inside the same area as the one represented in Figure 4.2, and visible in Figure 4.3. 


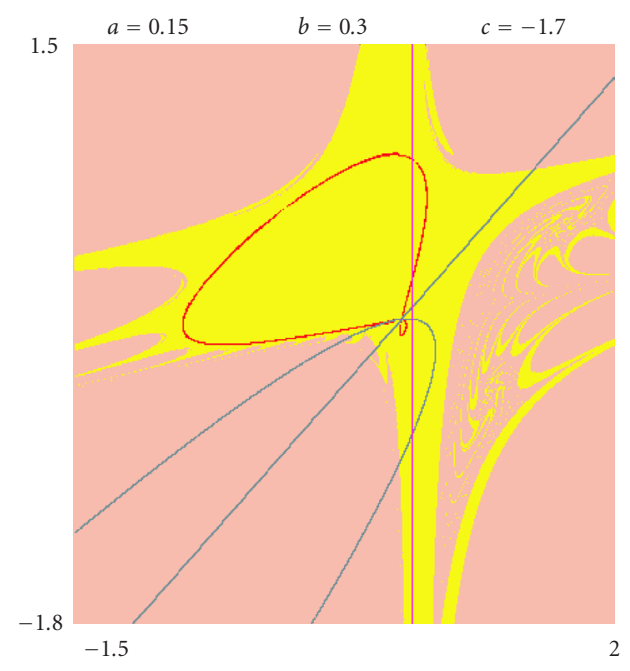

Figure 4.4. Formation of a loop after the crossing of the focal point.

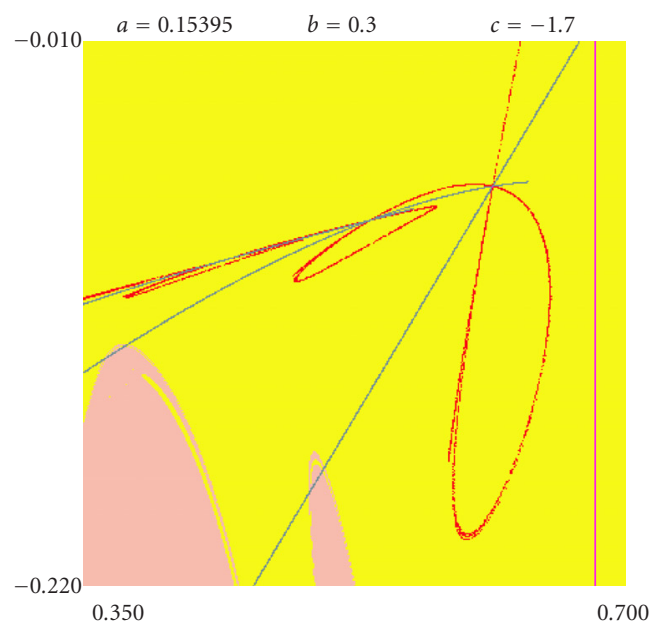

Figure 4.5. Contact between the focal point (and its images) and the attractor which becomes chaotic.

(3) For $a=0.133$, the attractor degenerates. It is a bifurcation value for which the focal point becomes a point cusp of the attractor before it degenerates. While increasing the value of the parameter $a$, loops on the attractor replace cusp points. The double points of these loops are confounded with the focal point and its images (Figures 4.4 and 4.5).

(II) We pose $a=-0.4, b=-0.3$, and we vary $c$.

(1) For $c=-0.1999$, the map $T$ has a saddle point of coordinates $(0,0)$, whose invariant stable manifold delimits the frontier of the basin of attraction of a stable focus of 


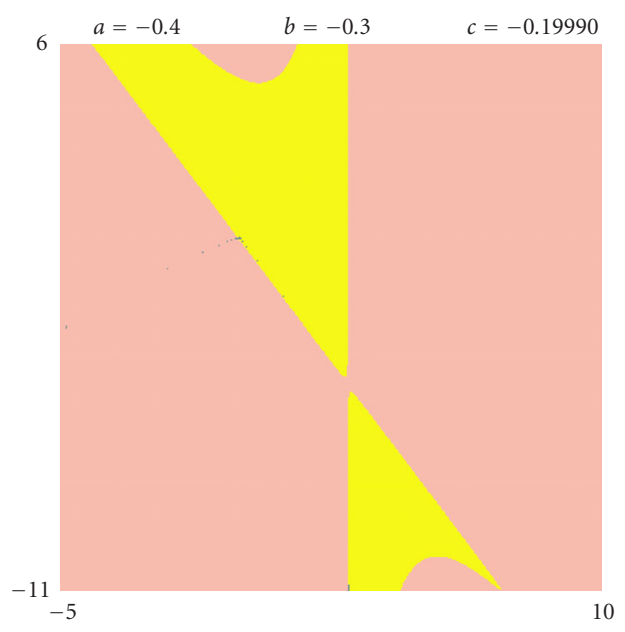

Figure 4.6. Basin nonconnected and iterates of focal point outside the basin.

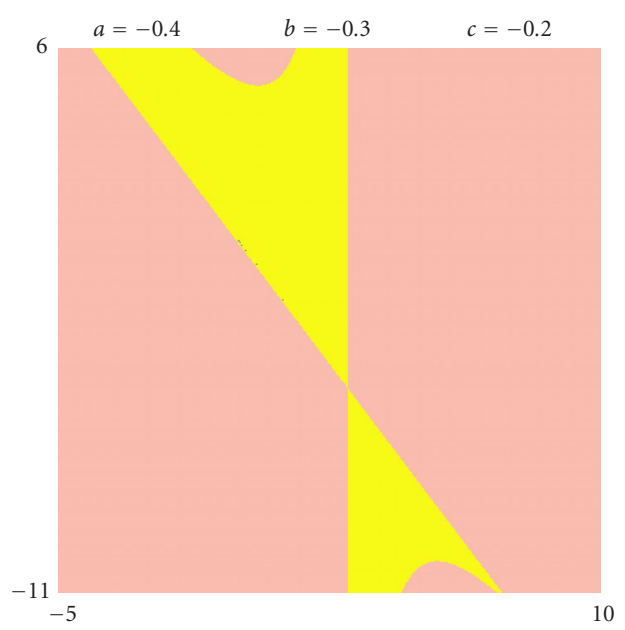

Figure 4.7. Iterates of focal point on the boundary of the basin.

coordinates $(1,0)$. We can see in Figure 4.6 that this basin of attraction is nonconnected and that the iterates, under $T$, of focal point $Q$ of $T^{-1}$, are outside the basin, which implies that $Q$ is outside the basin.

(2) For $c=-0.2000$, the focal point and its iterates by $T$ are on the border of the attraction basin of the stable focus and converge to the saddle (Figure 4.7). The basin of attraction is not connected, but its adherence is connected. 


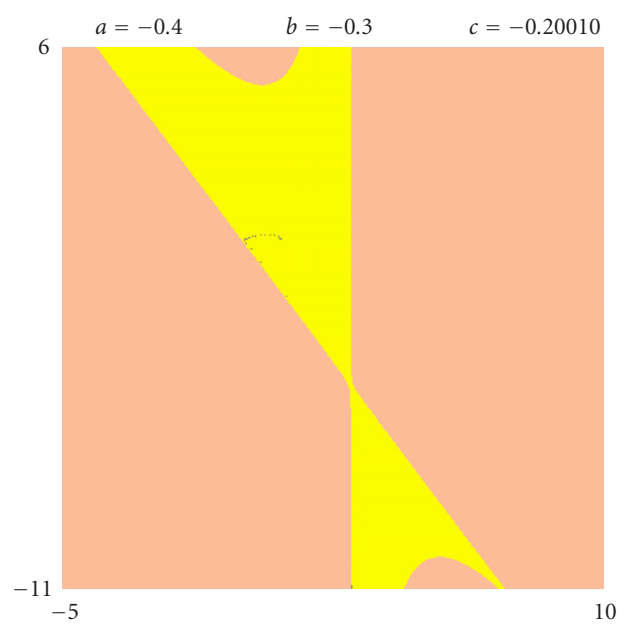

Figure 4.8. Basin connected and iterates of focal point inside the basin.

(3) For $c=-0.2001$, we can see in Figure 4.8 that the basin of attraction of the stable focus is connected and that iterates, under $T$, of focal point $Q$ of $T^{-1}$ are inside the basin, which implies that $Q$ is inside the basin.

Of these last three values of $c$, we conclude that $c=-0.2000$ is a basin bifurcation value, passing from nonconnected to connected and inversely, depending on whether the focal point is outside or inside the basin. It illustrates Proposition 3.1.

Whatever be the map with denominator, a contact of a attractor with a prefocal curve gives rise to a new type of bifurcation that causes the creation of cusp points and loops along the attractor which may give rise to a particular fractalization of this one.

\section{References}

[1] D. K. Arrowsmith, J. H. E. Cartwright, A. N. Lansbury, and C. M. Place, The Bogdanov map: bifurcations, mode locking, and chaos in a dissipative system, Internat. J. Bifur. Chaos Appl. Sci. Engrg. 3 (1993), no. 4, 803-842.

[2] G.-I. Bischi and L. Gardini, Basin fractalization due to focal points in a class of triangular maps, Internat. J. Bifur. Chaos Appl. Sci. Engrg. 7 (1997), no. 7, 1555-1577.

[3] - Focal points and basin fractalization in two classes of rational maps, Iteration theory (ECIT '96) (Urbino), Grazer Math. Ber., vol. 339, Karl-Franzens-University Graz, Graz, 1999, pp. 61-83.

[4] G.-I. Bischi, L. Gardini, and C. Mira, Plane maps with denominator. I. Some generic properties, Internat. J. Bifur. Chaos Appl. Sci. Engrg. 9 (1999), no. 1, 119-153.

[5] - Maps with a vanishing denominator. A survey of some results, Nonlinear Anal. 47 (2001), no. 4, 2171-2185.

[6] G.-I. Bischi and A. Naimzada, Global analysis of a nonlinear model with learning, Econ. Notes 26 (1997), no. 3, 143-174.

[7] I. Gumowski and C. Mira, Dynamique Chaotique. Translation ordre-desordre, Cepadues Éditions, Toulouse, 1980. 
[8] C. Mira, L. Gardini, A. Barugola, and J.-C. Cathala, Chaotic Dynamics in Two-Dimensional Noninvertible Maps, World Scientific Series on Nonlinear Science. Series A: Monographs and Treatises, vol. 20, World Scientific, New Jersey, 1996.

M. R. Ferchichi: Department of Mathematics, Faculty of Sciences, University of Annaba, BP 12, 23000 Annaba, Algeria

E-mail address: mrferchichi@yahoo.fr

I. Djellit: Department of Mathematics, Faculty of Sciences, University of Annaba, BP 12, 23000 Annaba, Algeria

E-mail address: a_djellit@hotmail.com 


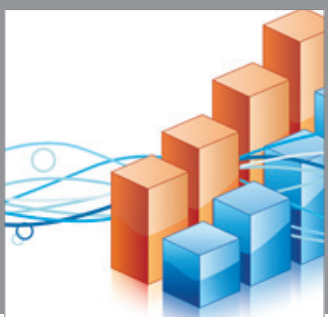

Advances in

Operations Research

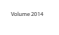

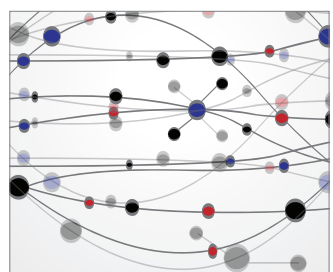

\section{The Scientific} World Journal
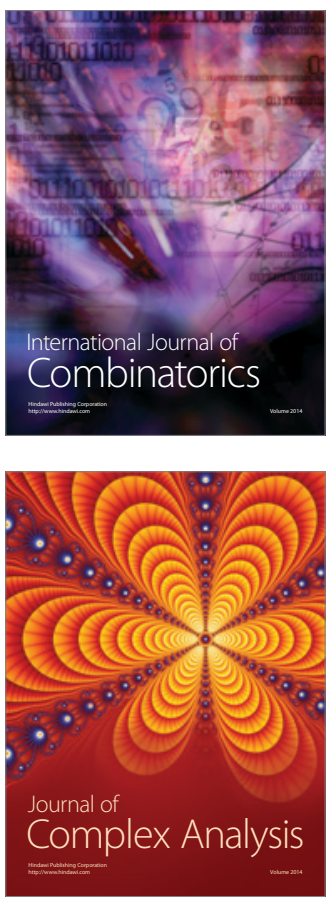

International Journal of

Mathematics and

Mathematical

Sciences
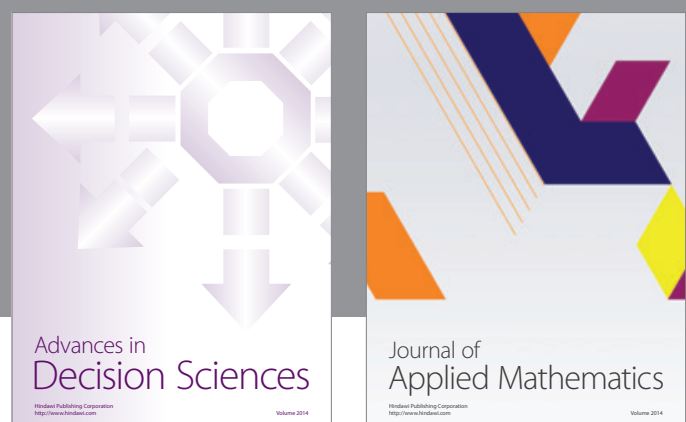

Journal of

Applied Mathematics
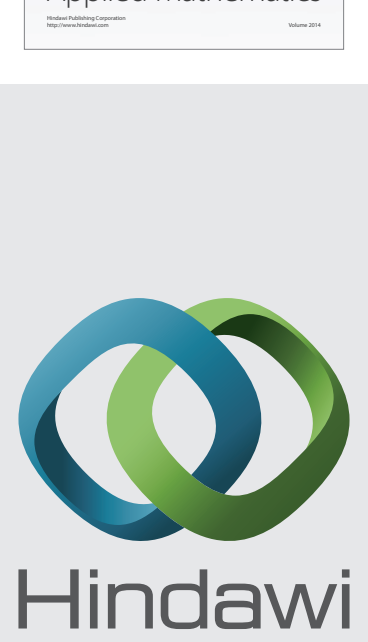

Submit your manuscripts at http://www.hindawi.com
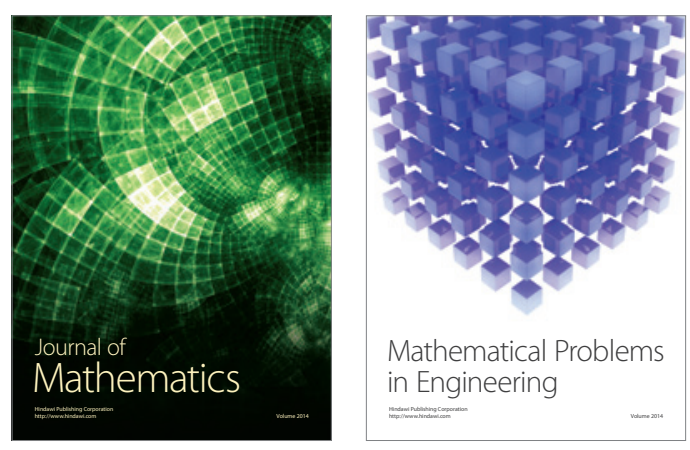

Mathematical Problems in Engineering
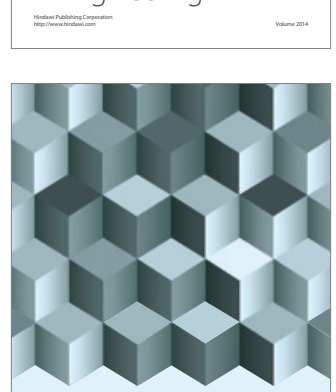

Journal of

Function Spaces
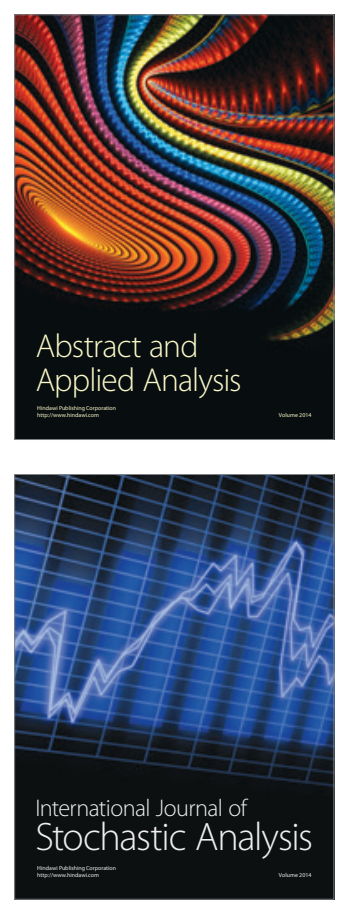

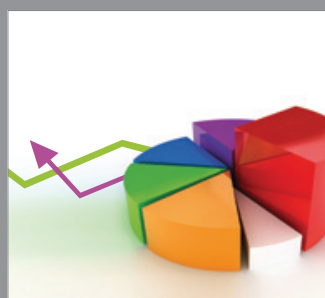

ournal of

Probability and Statistics

Promensencen
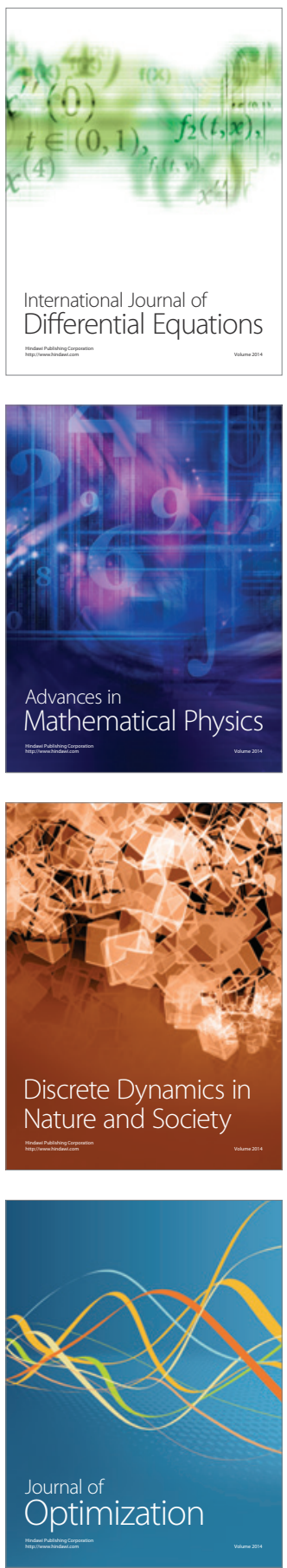